\title{
Adipose tissue trans-fatty acids and changes in body weight and waist circumference
}

\author{
Camilla P. Hansen ${ }^{1,2,3 *}$, Tina L. Berentzen ${ }^{3}$, Jane N. Østergaard ${ }^{1,2}$, Christina C. Dahm ${ }^{1}$, \\ Lars I. Hellgren ${ }^{4}$, Erik B. Schmidt ${ }^{2}$, Anne Tjønneland ${ }^{5}$, Thorkild I. A. Sørensen ${ }^{3,6}$, Kim Overvad ${ }^{1,2}$ \\ and Marianne U. Jakobsen ${ }^{1}$ \\ ${ }^{1}$ Section for Epidemiology, Department of Public Health, Aarhus University, Bartholins Allé 2, DK-800O Aarbus C, Denmark \\ ${ }^{2}$ Department of Cardiology, Center for Cardiovascular Research, Aalborg University Hospital, Aalborg, Denmark \\ ${ }^{3}$ Institute of Preventive Medicine, Bispebjerg and Frederiksberg Hospital, The Capital Region, Copenhagen, Denmark \\ ${ }^{4}$ Department of Systems Biology, Center for Biological Sequence Analysis, Technical University of Denmark, Lyngby, \\ Denmark \\ ${ }^{5}$ Danish Cancer Society Research Center, Copenhagen, Denmark \\ ${ }^{6}$ Novo Nordisk Foundation Center for Basic Metabolic Research, University of Copenhagen, Copenhagen, Denmark
}

(Submitted 24 June 2013 - Final revision received 7 October 2013 - Accepted 14 October 2013 - First published online 29 November 2013 )

\section{Abstract}

Previous studies have suggested that the intake of trans-fatty acids (TFA) plays a role in the development of obesity. The proportions of adipose tissue fatty acids not synthesised endogenously in humans, such as TFA, usually correlate well with the dietary intake. Hence, the use of these biomarkers may provide a more accurate measure of habitual TFA intake than that obtained with dietary questionnaires. The objective of the present study was to investigate the associations between the proportions of specific TFA in adipose tissue and subsequent changes in weight and waist circumference (WC). The relative content of fatty acids in adipose tissue biopsies from a random sample of 996 men and women aged 50-64 years drawn from a Danish cohort study was determined by GC. Baseline data on weight, WC and potential confounders were available together with information on weight and WC 5 years after enrolment. The exposure measures were total trans-octadecenoic acids $(18: 1 t), 18: 1 \Delta 6-10 t$, vaccenic acid $(18: 1 \Delta 11 t)$ and rumenic acid $(18: 2 \Delta 9 c, 11 t)$. Data were analysed using multiple regression with cubic spline modelling. The median proportion of total adipose tissue $18: 1 t$ was $1 \cdot 52 \%(90 \%$ central range $0 \cdot 98,2 \cdot 19)$ in men and $1.47 \%(1 \cdot 01,2 \cdot 19)$ in women. No significant associations were observed between the proportions of total 18: $1 t, 18: 1 \Delta 6-10 t$, vaccenic acid or rumenic acid and changes in weight or WC. The present study suggests that the proportions of specific TFA in adipose tissue are not associated with subsequent changes in weight or WC within the exposure range observed in this population.

\section{Key words: Trans-fatty acids: Adipose tissue: Weight: Waist circumference}

Previous studies have suggested that trans-fatty acids (TFA) play a role in the development of obesity ${ }^{(1)}$. TFA are unsaturated fatty acids with at least one double bond in the trans-configuration, and they are formed either by industrial hydrogenation or by biohydrogenation in ruminants. TFA from both industrial and ruminant sources mainly contain trans-isomers of octadecenoic acid (18:1), but the distribution of isomers differs between the two sources ${ }^{(2)}$. In industrially partially hydrogenated oils, elaidic acid $(18: 1 \Delta 9 t)$ is the most abundant TFA, but $18: 1 \Delta 10 t$ is also present in relatively large amounts. In contrast, dairy and meat products from ruminants mainly contain vaccenic acid $(18: 1 \Delta 11 t)$. In addition, rumenic acid $(18: 2 \Delta 9 c, 11 t)$, an isomer of conjugated linoleic acid (CLA), is present in TFA from ruminant sources. Vaccenic acid can also be converted into rumenic acid endogenously in humans $^{(3,4)}$. Several animal studies have shown that feeding CLA reduces body weight and fat depositions, but the results from human studies are inconsistent ${ }^{(5,6)}$.

Positive associations between total reported intake of TFA and changes in weight and waist circumference (WC) have been found by two large American cohort studies ${ }^{(7,8)}$. In a previous study, we observed a weak inverse association of ruminant TFA intake with weight change, but not with WC change $^{(9)}$. A cross-sectional study of the associations between

Abbreviations: CLA, conjugated linoleic acid; TFA, trans-fatty acids; WC, waist circumference; WC $\mathrm{BMI}_{\mathrm{B}}$, changes in waist circumference adjusted for concurrent changes in BMI; Weight ${ }_{\mathrm{WC}}$, changes in weight adjusted for concurrent changes in waist circumference.

*Corresponding author: C. P. Hansen, fax +453816 3119, email cph@soci.au.dk 
adipose tissue TFA and different anthropometric measures has suggested that the individual TFA may have divergent effects on adiposity ${ }^{(10)}$.

Biomarkers of the dietary intake of TFA may provide a more accurate measure of habitual TFA intake than that obtained with dietary questionnaires ${ }^{(11)}$. The content of fatty acids in adipose tissue reflects the dietary intake of fatty acids during the last couple of years ${ }^{(12)}$, and the proportions of adipose tissue fatty acids not synthesised endogenously in humans, such as TFA, tend to correlate well with the reported habitual dietary intake ${ }^{(13,14)}$.

In epidemiological studies of TFA and CVD, the intake of TFA from industrial sources has been shown to be associated with higher risks, whereas that of TFA from ruminant sources did not exhibit such an association ${ }^{(15,16)}$. This may be due to different biological effects of specific TFA isomers or to a threshold effect of TFA, since TFA of industrial origin are usually consumed in much greater amounts than TFA from ruminant sources.

The aim of the present study was to investigate the associations between the proportions of specific TFA in adipose tissue and subsequent changes in body weight and WC.

\section{Materials and methods}

\section{Study population and design}

From December 1993 to May 1997, all men and women, born in Denmark, aged 50-64 years, living in the greater Aarhus or Copenhagen areas, and with no previous cancer diagnosis registered in the Danish Cancer Registry were invited to participate in the Danish Diet, Cancer and Health study ( $n$ 160 725). In total, 57053 (35\%) participants were recruited, and for the present study, a random sample of 1869 cohort members was drawn using simple random sampling.

At baseline, the participants completed a FFQ about their dietary intake during the previous year and a self-administered questionnaire about lifestyle and sociodemographic factors and health. The participants underwent a physical examination at the study clinics in Aarhus or Copenhagen, and biological samples were collected. Follow-up information was collected through postal questionnaires between September 1999 and October 2002. The Danish Data Protection Agency and the regional Ethics Committee approved the study, which was in accordance with the Helsinki Declaration II, and the participants gave written consent before enrolment in the study. The study design and measurement procedures have been described in detail elsewhere ${ }^{(17)}$

\section{Adipose tissue analysis}

A $35-50 \mathrm{mg}$ adipose tissue biopsy was taken from the buttocks of the participants at enrolment using a luerlock system (Terumo, Terumo Corporation) consisting of a needle, a Venoject multisample luer adaptor and an evacuated blood tube, according to the method of Beynen \& Katan ${ }^{(18)}$, and stored in liquid $\mathrm{N}_{2}$ vapour (maximum $-150^{\circ} \mathrm{C}$ ) in the original needle and sample adapter until analysis. The biopsies taken from participants in the random sample were thawed, and approximately $2-4 \mathrm{mg}$ were put into a glass and prewarmed at $50^{\circ} \mathrm{C}$ for $10 \mathrm{~min}$. Subsequently, the fat was dissolved in heptane at $50^{\circ} \mathrm{C}$, and the fatty acids were transesterified using $2 \mathrm{M}-\mathrm{KOH}$ in methanol at $50^{\circ} \mathrm{C}$ for $2 \mathrm{~min}$, according to International Union of Pure and Applied Chemistry (IUPAC) Standard Methods for Analysis of Oils, Fats and Derivatives. The composition of fatty acids was analysed by GC using a Varian 3900 GC with a CP-8400 autosampler (Varian) equipped with a flame ionisation detector. Split injection mode (injector temperature $280^{\circ} \mathrm{C}$ and split ratio 1:20), a CP-sil 88, $60 \mathrm{~m} \times 0.25 \mathrm{~mm}$ internal diameter capillary column (Varian), temperature programming from 90 to $210^{\circ} \mathrm{C}$, increasing with $6^{\circ} \mathrm{C} / \mathrm{min}$, and constant flow were used. Helium was used as the carrier gas. Commercially available standards (Nu-chek-Prep, Inc.) were used to identify the individual fatty acids. This approach permits the quantification of fatty acid methyl esters with twelve to twenty-four carbon atoms and the separation and quantification of several TFA. The content of fatty acids is expressed as percentages of total fatty acids (wt $\%$ ). The following specific TFA were determined: trans-6 (+trans-8)-18:1 (18:1 $\Delta 6 t(+\Delta 8 t))$; trans-9-18: 1 (18:1 $\Delta 9 t$ ) (elaidic acid); trans-10-18:1 (18:1 $\Delta 10 t)$; trans-11-18:1 (18:1 $\Delta 11 t)$ (vaccenic acid); cis-9, trans-11-18:2 (18:2 $\Delta 9 c, 11 t)$ (rumenic acid). The interassay CV for the determination of the trans-18:1 positional isomers were between $3 \cdot 7 \%$ (elaidic acid) and $7 \cdot 1 \%$ (vaccenic acid), and the inter-assay $\mathrm{CV}$ for the determination of rumenic acid was $2.9 \%$.

The fatty acids 18:1 $\Delta 6 t(+\Delta 8 t), 18: 1 \Delta 9 t$ and 18:1 $\Delta 10 t$ share the same main dietary source (industrially partially hydrogenated oils), and the pairwise correlations between them were between 0.90 and 0.92 . Therefore, we decided to merge these positional TFA isomers into one exposure category labelled 18:1 $\Delta 6-10 t$. In contrast, the main dietary source for the fatty acid 18:1 $\Delta 11 t$ is ruminant products, and the correlations between this fatty acid and the other $18: 1$ trans-isomers were much lower $(0 \cdot 42-0 \cdot 55)$. Total trans$18: 1(18: 1 t)$ was defined as $18: 1 \Delta 6-10 t+18: 1 \Delta 11 t$.

\section{Anthropometric measures}

Baseline measurements of height, weight and WC were taken by laboratory technicians. Height was measured with the participants standing without shoes and recorded to the nearest $0.5 \mathrm{~cm}$, and weight was measured with a digital scale with the participants wearing light underwear and recorded to the nearest $100 \mathrm{~g}$. WC was measured at the narrowest circumference between the lower rib and the iliac crest (the natural waist) or, if waist narrowing was indeterminable, halfway between the lower rib and the iliac crest and recorded to the nearest $0.5 \mathrm{~cm}$. Follow-up weight and $\mathrm{WC}$ were selfreported. A measuring tape was provided with the postal questionnaires and the participants were instructed to measure their WC at the umbilicus level.

The outcomes in the present study were defined as average annual changes in body weight and WC, which were calculated by subtracting the baseline values from the follow-up 
values and dividing these differences by the duration of follow-up time. In addition, we studied changes in WC for given changes in $\mathrm{BMI}\left(\mathrm{WC}_{\mathrm{BMI}}\right)$ defined as a linear regression model with change in WC as the outcome and concurrent change in BMI included as a covariate. Similarly, we also studied changes in weight adjusted for concurrent changes in WC (Weight $\left.\mathrm{WC}_{\mathrm{C}}\right)$.

\section{Exclusion criteria}

Participants who had incomplete questionnaires with multiple errors and participants for whom data on follow-up time, adipose tissue TFA, weight, WC or any of the potential confounders were missing were excluded (Fig. 1). Furthermore, participants with relative changes in weight or WC below the sex-specific 1st percentile or above the 99th percentile were excluded due to potential measurement errors. Individuals diagnosed with chronic diseases might change their dietary habits and there may be alterations in their body composition as a result of these changes or as a result of the disease itself. Therefore, participants who had one of the following chronic diseases at baseline or at follow-up were excluded: cancer; CVD; chronic obstructive pulmonary disease; diabetes. Diagnoses of cancer were identified by record linkage to the Danish Cancer Registry ${ }^{(19)}$. Diagnoses of CVD and chronic obstructive pulmonary disease were

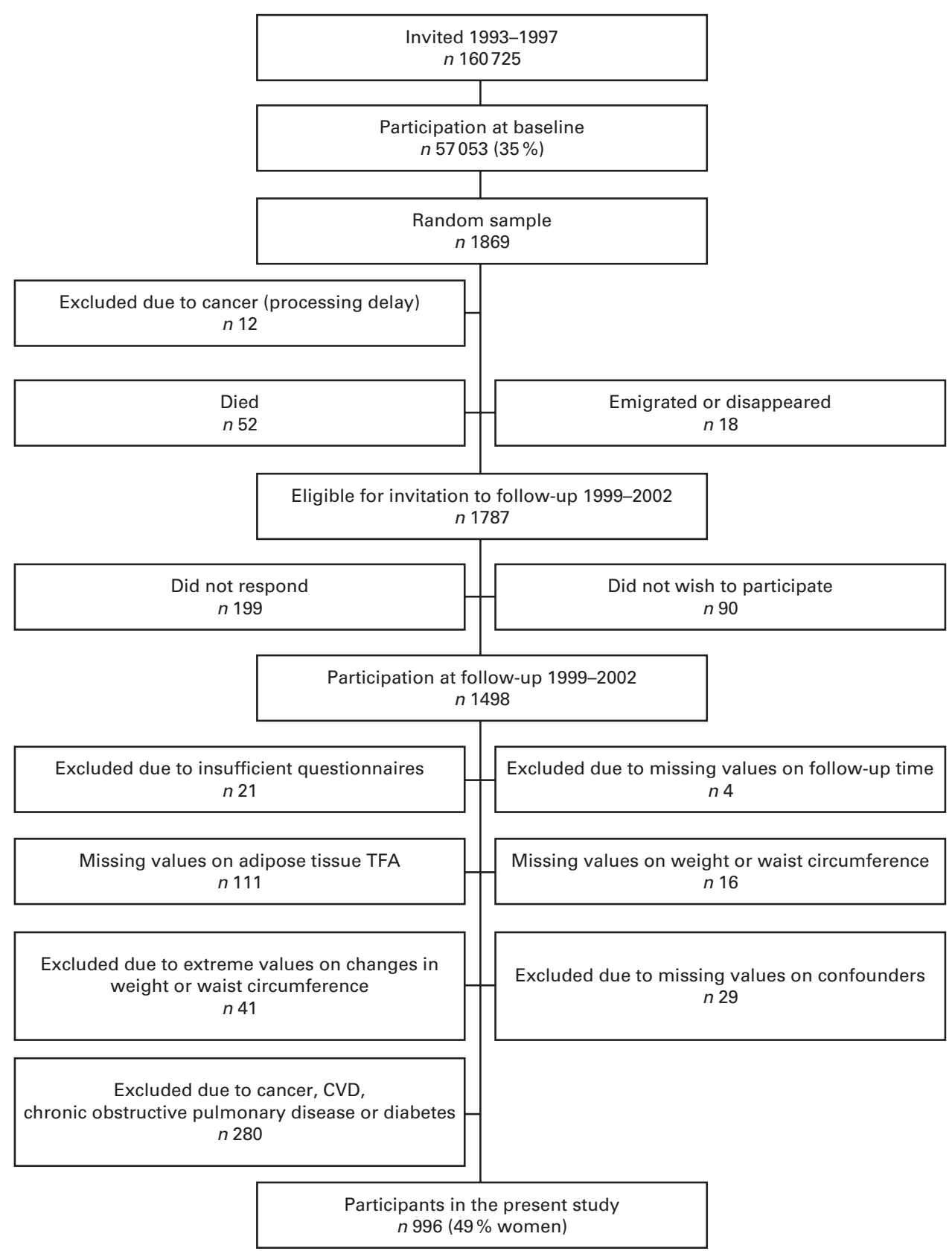

Fig. 1. Flow chart of the study population. TFA, trans-fatty acids. 
identified by record linkage to the Danish National Patient Registry $^{(20)}$. For diabetes, diagnoses were either self-reported or identified by record linkage to the Danish National Diabetes Register $^{(21)}$.

\section{Statistical analyses}

The associations between the proportions of adipose tissue TFA and average annual changes in weight, $\mathrm{WC}, \mathrm{WC}_{\mathrm{BMI}}$ and Weight $_{\mathrm{WC}}$ were studied using multiple linear regression. Analyses were carried out for all the participants and separately for each sex. The following variables were included as covariates in the model: sex; age; baseline BMI; change in BMI (only in the analysis of change in $\mathrm{WC}_{\mathrm{BMI}}$ ); baseline $\mathrm{WC}$ (only in the analyses of changes in $\mathrm{WC}, \mathrm{WC}_{\mathrm{BMI}}$ and $\mathrm{Weight}_{\mathrm{WC}}$ ); change in WC (only in the analysis of change in Weight $\mathrm{WC}_{\mathrm{C}}$ ); smoking (never, former, current $<15 \mathrm{~g} / \mathrm{d}$, current $15-25 \mathrm{~g} / \mathrm{d}$, current $>25 \mathrm{~g} / \mathrm{d}$ ); alcohol intake $(\mathrm{g} / \mathrm{d})$; sports activity (h/week); education ( 7 years or less, $8-10$ years, more than 10 years); menopausal status (in women; pre, post, unknown); current use of hormone replacement therapy (in women; yes, no). The fatty acid variables 18:1 $\Delta 6-10 t, 18: 1 \Delta 11 t$ and 18:2
$\Delta 9 c, 11 t$ were also analysed in a mutually adjusted model containing the potential confounders included in the main model.

All the continuous covariates were included as restricted cubic splines with three knots at the 10th, 50th and 90th percentiles $^{(22,23)}$. The data were modelled using restricted cubic splines to be able to observe non-linear and threshold effects of TFA. Associations were considered to be significant if at least one of the spline parameters differed significantly from zero assessed by the Wald test. The associations between the proportions of adipose tissue TFA and changes in anthropometry were considered to be non-linear if the last spline parameter differed significantly from zero assessed by the Wald test. If the test of non-linearity was rejected, linear regression coefficients for the associations between the proportions of adipose tissue TFA and changes in anthropometry were provided. Two-sided $P$ values below 0.05 were considered to be statistically significant.

Effect modification by sex was formally tested using a likelihood ratio test of nested models with and without the relevant product terms. The observed associations in men and women did not differ substantially, and none of the tests was statistically significant. Therefore, only results from the analyses of all the participants are presented.

Table 1. Characteristics of the study population (Median values with 5th and 95th percentiles)

\begin{tabular}{|c|c|c|c|c|}
\hline & \multicolumn{2}{|r|}{ Men $(n 512)$} & \multicolumn{2}{|c|}{ Women ( $n$ 484) } \\
\hline & Median & 5th and 95th percentile & Median & 5th and 95th percentile \\
\hline Age (years) & $55 \cdot 6$ & $50 \cdot 8-64 \cdot 2$ & $55 \cdot 5$ & $50 \cdot 7-63.5$ \\
\hline Weight at baseline $(\mathrm{kg})$ & $81 \cdot 2$ & $66 \cdot 3-103 \cdot 7$ & $65 \cdot 8$ & $52 \cdot 8-87.5$ \\
\hline Annual change in weight (g) & 73 & $-1151-1288$ & -19 & $-1253-1098$ \\
\hline WC at baseline & 95 & $82-110$ & 78 & $67-100$ \\
\hline Annual change in WC $(\mathrm{cm})$ & 0.6 & $-1 \cdot 2-2 \cdot 3$ & 1.3 & $-0.8-3.5$ \\
\hline Height (m) & 1.76 & $1.66-1.88$ & 1.65 & $1.55-1.74$ \\
\hline BMI at baseline $\left(\mathrm{kg} / \mathrm{m}^{2}\right)$ & $26 \cdot 2$ & $22 \cdot 0-31 \cdot 7$ & $24 \cdot 3$ & $19 \cdot 8-32 \cdot 0$ \\
\hline \multicolumn{5}{|c|}{ Adipose tissue TFA ( $\%$ of fatty acids) } \\
\hline Total trans-18: 1 & 1.52 & $0.98-2 \cdot 19$ & 1.47 & $1.01-2 \cdot 19$ \\
\hline $18: 1 \Delta 6-10 t$ & $1 \cdot 17$ & $0.72-1.74$ & $1 \cdot 13$ & $0.74-1.71$ \\
\hline $18: 1 \Delta 6(+\Delta 8 t)$ & 0.29 & $0.17-0.46$ & 0.29 & $0.18-0.45$ \\
\hline $18: 1 \Delta 9 t$ & 0.55 & $0.34-0.84$ & 0.52 & $0.34-0.81$ \\
\hline $18: 1 \Delta 10 t$ & 0.33 & $0.21-0.47$ & 0.32 & $0.22-0.45$ \\
\hline $18: 1 \Delta 11 t$ & 0.31 & $0.18-0.43$ & 0.29 & $0.18-0.43$ \\
\hline $18: 2 \Delta 9 c, 11 t$ & 0.43 & $0.29-0.57$ & 0.46 & $0.32-0.63$ \\
\hline Sports activity (h/week) & 1 & $0-6$ & 1 & $0-4$ \\
\hline \multicolumn{5}{|l|}{ Smoking (\%) } \\
\hline Never & \multicolumn{2}{|r|}{30} & \multicolumn{2}{|r|}{46} \\
\hline Former & & 36 & & 22 \\
\hline Current & \multicolumn{2}{|r|}{$\begin{array}{l}36 \\
34\end{array}$} & \multicolumn{2}{|r|}{32} \\
\hline Alcohol intake (g/d) & $20 \cdot 4$ & $2 \cdot 2-71 \cdot 4$ & $9 \cdot 8$ & $0 \cdot 8-39 \cdot 3$ \\
\hline \multicolumn{5}{|l|}{ Education (\%) } \\
\hline$\leq 7$ years & \multicolumn{2}{|r|}{$\begin{array}{l}30 \\
42\end{array}$} & \multicolumn{2}{|r|}{29} \\
\hline $8-10$ years & \multicolumn{2}{|r|}{$\begin{array}{l}42 \\
28\end{array}$} & \multicolumn{2}{|r|}{52} \\
\hline$>10$ years & \multirow{2}{*}{\multicolumn{2}{|c|}{28}} & \multicolumn{2}{|r|}{19} \\
\hline \multicolumn{3}{|l|}{ Menopausal status (\%) } & & \\
\hline Pre & \multicolumn{2}{|r|}{-} & \multicolumn{2}{|r|}{20} \\
\hline Post & \multicolumn{2}{|r|}{ - } & \multicolumn{2}{|r|}{58} \\
\hline Unknown & \multicolumn{2}{|r|}{-} & \multicolumn{2}{|r|}{22} \\
\hline \multicolumn{5}{|l|}{ Use of HRT (\%) } \\
\hline No & \multirow{2}{*}{\multicolumn{2}{|c|}{ - }} & \multicolumn{2}{|r|}{69} \\
\hline Yes & & - & \multicolumn{2}{|r|}{31} \\
\hline
\end{tabular}

WC, waist circumference; TFA, trans-fatty acids; HRT, hormone replacement therapy. 
Residual $v$. fitted plots were used to check the assumption of homogeneity of variance. Histograms and $\mathrm{P}-\mathrm{P}$ plots of the residuals for each model were used to check the assumption of normally distributed error terms. No violations were found.

Data analyses were carried out using the Stata statistical software, version 11.2 (Stata Corporation).

\section{Results}

A flow chart of the study population is shown in Fig. 1. Among the 1869 subjects sampled at baseline, twelve were later excluded due to a cancer diagnosis before recruitment, which, due to processing delays, had not been registered in the Danish Cancer Registry at the time of invitation. Between baseline and follow-up, seventy individuals died or were lost to follow-up and 289 individuals did not respond or did not wish to participate at the follow-up. Among the 1498 participants who participated at follow-up, exclusions were made according to the criteria described earlier. This left 996 subjects free from major illnesses and with complete data on exposures, outcomes and potential confounders to be included in the analyses.
The characteristics of the study participants are given in Table 1 . The median proportion of total adipose tissue $18: 1 t$ was $1.52 \%(90 \%$ central range $0.98,2 \cdot 19)$ in men and $1.47 \%$ (90\% central range $1.01,2.19)$ in women. The median annual weight change was $73 \mathrm{~g}$ (90\% central range -1151 , 1288) in men and $-19 \mathrm{~g}$ (90\% central range -1253 , 1098) in women, whereas the median annual WC change was $0.6 \mathrm{~cm}(90 \%$ central range $-1 \cdot 2,2.3)$ in men and $1.3 \mathrm{~cm}$ (90\% central range $-0 \cdot 8,3 \cdot 5)$ in women.

\section{Change in weight}

No significant associations were observed between the adipose tissue levels of total $18: 1 t$ or $18: 1 \Delta 6-10 t$ and changes in weight ( $P=0 \cdot 11$ and $P=0 \cdot 10$, respectively) (Fig. 2). Similarly, no significant results were obtained for vaccenic acid or rumenic acid ( $P=0.48$ and $P=0.46$, respectively) (Fig. 2). The analysis of 18:1 $\Delta 6-10 t$, vaccenic acid and rumenic acid in a mutually adjusted model did not change the observed associations substantially (data not shown). None of the tests of non-linearity was statistically significant, and linear regression coefficients for the associations between the proportions of adipose tissue TFA and changes in weight are given in Table 2 .
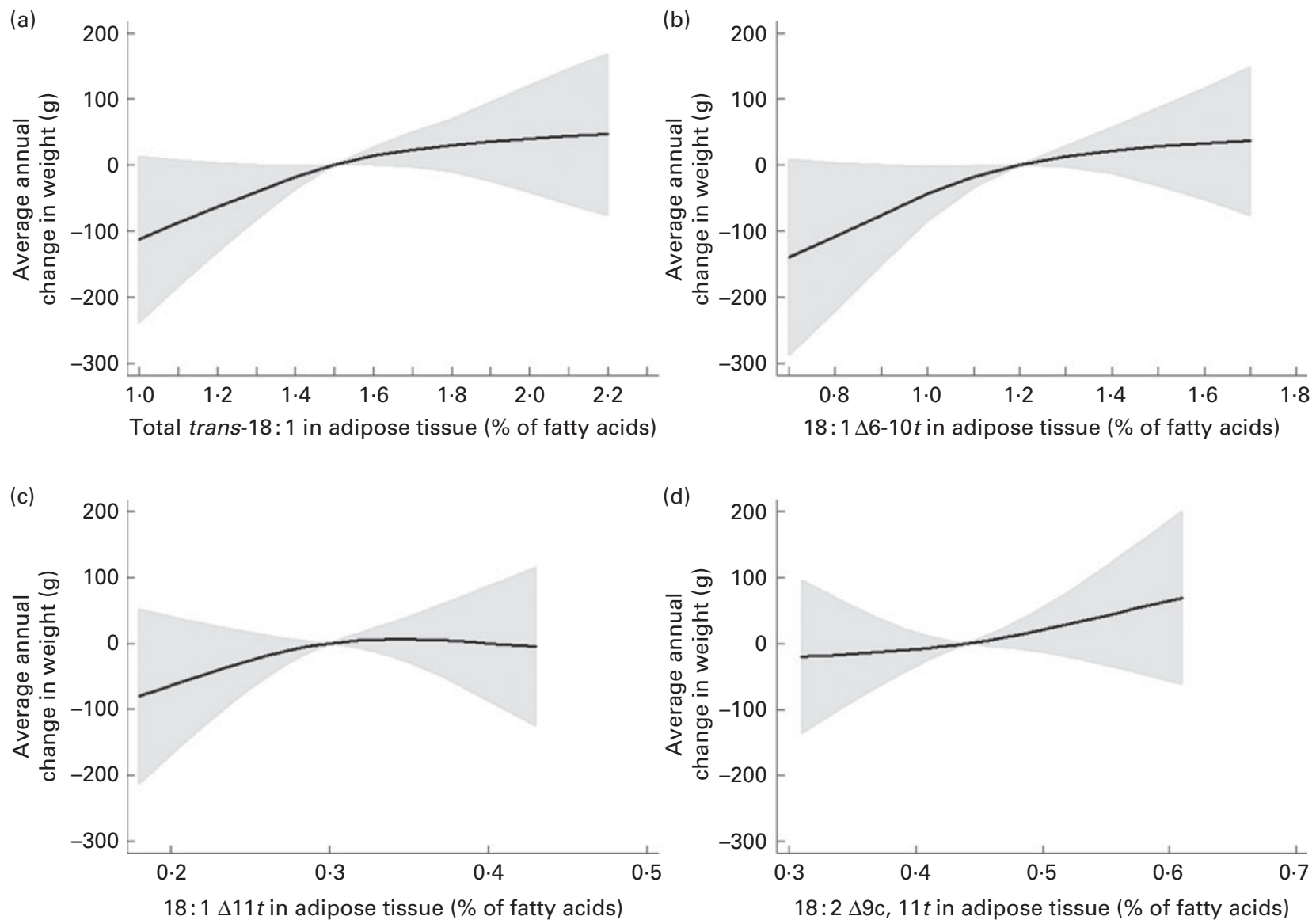

Fig. 2. Proportion of adipose tissue trans-fatty acids and average annual change in body weight. The solid lines are the exposure variables modelled with restricted cubic splines with three knots and the shaded areas are the $95 \% \mathrm{Cl}$. Adjustments: sex; age; baseline BMl; smoking; alcohol intake; sports activity; education; menopausal status (only women); current use of hormone replacement therapy (only women). $P$ values from the test of association: (a) total trans-18: 1 $(P=0.11)$; (b) 18:1 $\Delta 6-10 t(P=0 \cdot 10)$; (c) vaccenic acid $(P=0.48)$; (d) rumenic acid $(P=0.46)$. 
Table 2. Linear associations between the proportions of adipose tissue trans-fatty acids (TFA, \% of fatty acids) and changes in weight (kg/year) and waist circumference (cm/year)

( $\beta$-Coefficients and $95 \%$ confidence intervals)

\begin{tabular}{lccccc}
\hline & \multicolumn{2}{c}{ Weight change } & & \multicolumn{2}{c}{$\begin{array}{c}\text { Waist circumference } \\
\text { change }\end{array}$} \\
\cline { 2 - 3 } \cline { 5 - 6 } & $\beta^{*}$ & $95 \% \mathrm{Cl}$ & & $\beta^{*}$ & $95 \% \mathrm{Cl}$ \\
\hline Total trans-18:1 & 0.126 & $-0.006,0.257$ & & 0.10 & $-0.11,0.32$ \\
$18: 1 \Delta 6-10 t$ & 0.153 & $-0.004,0.310$ & & 0.11 & $-0.15,0.36$ \\
$18: 1 \Delta 11 t$ & 0.268 & $-0.339,0.876$ & & 0.28 & $-0.71,1.27$ \\
$18: 2 \Delta 9 c, 11 t$ & 0.297 & $-0.194,0.787$ & & 0.34 & $-0.46,1.13$
\end{tabular}

${ }^{*} \beta$-coefficient for $1 \%$ point difference in the percentage of TFA in adipose tissue. Adjustments: sex; age; baseline BMI; baseline waist circumference (WC, only in the analysis of WC change); smoking; alcohol intake; sports activity; education; menopausal status (only women); current use of hormone replacement therapy (only women).

\section{Changes in weight adjusted for concurrent changes in} waist circumference

Neither the adipose tissue level of total 18:1t nor that of any of the specific TFA was significantly associated with changes in Weight ${ }_{\mathrm{WC}}$ (Fig. S1 and Table S1, available online).
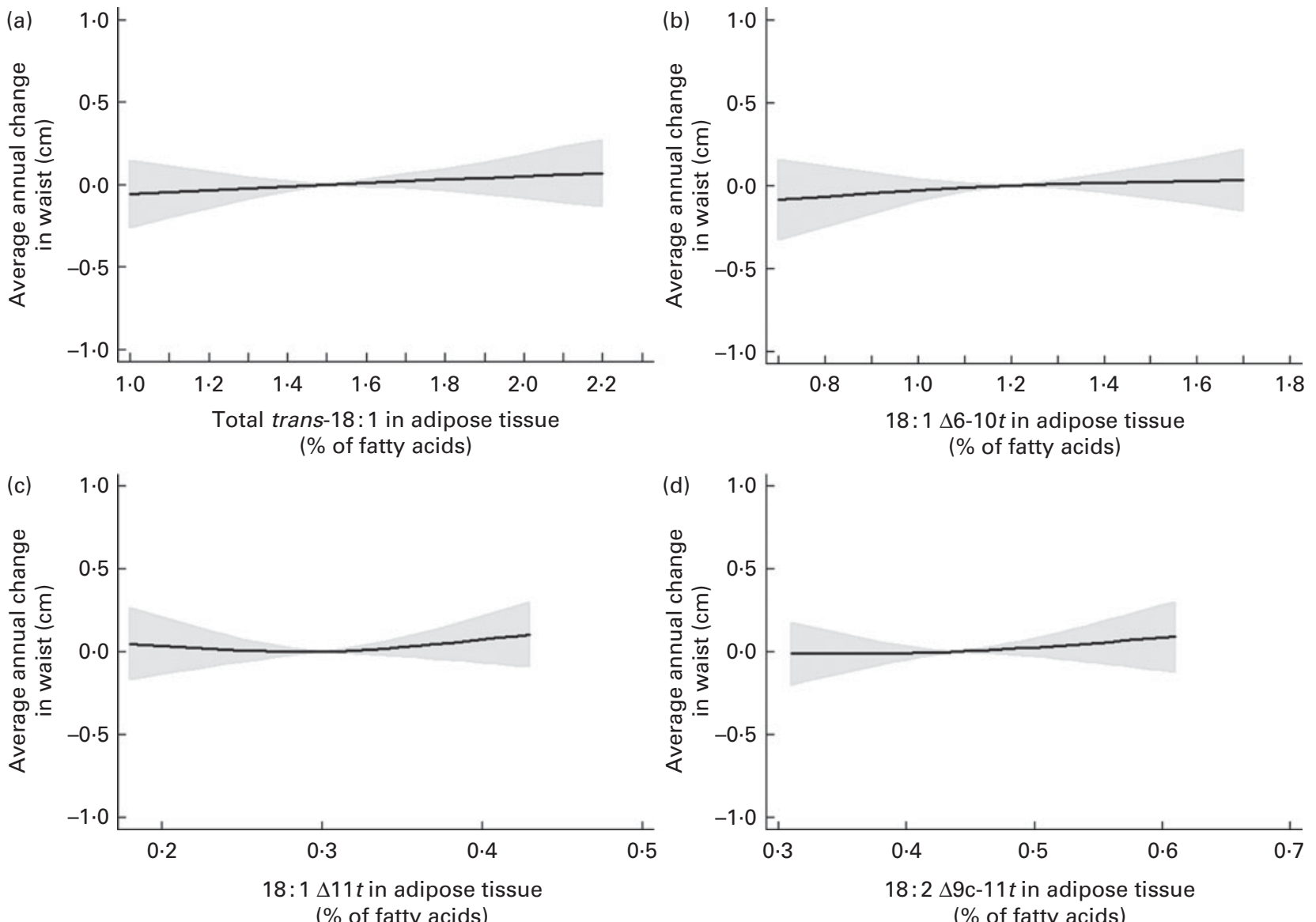

\section{changes in BMI}

Neither the adipose tissue level of total 18:1t nor that of any of the specific TFA was significantly associated with changes in $\mathrm{WC}_{\mathrm{BMI}}$ (Fig. S2 and Table S1, available online)

\section{Discussion}

In the present study, the proportions of TFA in adipose tissue did not predict subsequent changes in anthropometry.

Fig. 3. Proportions of adipose tissue trans-fatty acids and average annual change in waist circumference. The solid lines are the exposure variables modelled with restricted cubic splines with three knots and the shaded areas are the $95 \% \mathrm{Cl}$. Adjustments: sex; age; baseline BMI; baseline waist circumference; smoking; alcohol intake; sports activity; education; menopausal status (only women); current use of hormone replacement therapy (only women). $P$ values from the test of association: (a) total trans-18:1 ( $P=0.63)$; (b) 18:1 $\Delta 6-10 t(P=0.68)$; (c) vaccenic acid $(P=0.58)$; (d) rumenic acid $(P=0.66)$. 
The study population was a random sample of the Danish Diet, Cancer and Health cohort. Loss to follow-up was mainly due to non-response. Non-participants at follow-up did not differ from the participants with regard to age and adipose tissue levels of TFA, but non-participants tended to have slightly higher baseline BMI and WC than the participants. Thus, non-response may be related to changes in anthropometry, but not to both the exposure to TFA and changes in anthropometry. Therefore, selection bias is of limited concern.

The proportions of adipose tissue fatty acids not synthesised endogenously in humans, such as TFA, are considered as biomarkers of the dietary intake from the past several months to more than a year ${ }^{(13,24)}$. Thus, the proportions of TFA in adipose tissue are an objective measure of long-term endogenous exposure to TFA. Published correlation coefficients between TFA intake (as percentage of total fat) estimated from FFQ and the proportion of adipose tissue TFA have varied from $0 \cdot 29$ to $0 \cdot 67^{(25-29)}$. In the present study, the median proportion of adipose tissue elaidic acid was $0.55 \%$, whereas that of vaccenic acid was $0.31 \%$, indicating that the main dietary source of TFA in the study population was industrially partially hydrogenated oils at the time the adipose tissue biopsies were collected. The intake of TFA from industrial sources declined from $2 \cdot 2 \mathrm{~g} / \mathrm{d}$ in 1992 to $0.4 \mathrm{~g} / \mathrm{d}$ in 1999 in the Danish population $^{(30)}$. In 2004 , a restriction on the content of industrially produced TFA in fats was imposed in Denmark, and as a result, TFA from industrial sources no longer contribute substantially to the intake of TFA in the Danish population ${ }^{(30)}$. Although a change in dietary intake during follow-up is expected to cause changes in the proportions of specific TFA in adipose tissue, the study participants are presumed to be ranked correctly according to their TFA exposure. The adipose tissue biopsies were sampled from the buttocks of the participants in the present study. The composition of fatty acids in adipose tissue varies between depots ${ }^{(13)}$, and in a study conducted among children, a higher content of TFA has been observed in abdominal fat depots than in buttock fat depots ${ }^{(31)}$. However, the reported differences in the composition of fatty acids between fat depots are rather small $^{(13,31)}$. Baseline anthropometric measures were obtained by trained staff, whereas follow-up weight and WC were self-reported. A validation study within the Diet, Cancer and Health cohort has shown that self-reported WC at the umbilicus level is usable as a proxy for technician-measured WC at the natural waist in regression analyses if baseline BMI and WC are included as covariates ${ }^{(32)}$. An overall net weight loss was reported among women in the present study. This may be due to the age of the study population or due to under-reporting of follow-up weight. Most probably, the reporting of follow-up weight and WC was not related to the proportions of TFA in adipose tissue. Thus, systematic measurement errors are unlikely to have affected the results. However, random measurement errors could potentially have biased the results towards null.

Adjustment for potential confounders had no notable influence on the observed associations. Furthermore, adjusting for the continuous confounders using restricted cubic splines with five knots instead of three knots did not change the observed associations. Although residual confounding cannot be eliminated, these sensitivity analyses support that it may not be of major concern. However, confounders not taken into account may have affected the results. The metabolism of fatty acids and thus their concentrations in body tissue may be affected by lifestyle. A study of the adipose tissue content of $14: 0,15: 0$ and $17: 0$ as a biomarker of dairy fat intake has suggested that adjustments should be made for alcohol intake and physical activity when these fatty acids are used as biomarkers of dairy fat intake ${ }^{(33)}$. This may also apply to adipose tissue TFA. In the present study, both alcohol intake and physical activity were included as covariates. Previous studies have found inverse associations between the proportions of $14: 0,15: 0$ and 17:0 in adipose tissue and weight, BMI and $\mathrm{WC}^{(34-36)}$. However, further adjustment for 15:0 and 17:0 did not change the observed association for vaccenic acid (data not shown). The content of specific adipose tissue fatty acids is expressed as proportions of total adipose tissue fatty acids. When adjustment is made for other fatty acids, either as proportions in adipose tissue or as those from dietary intake, food sources contributing to variation in the specific TFA are restricted to food sources that do not contribute to variation in the fatty acids that are controlled for. Thus, mutual adjustment for several specific adipose tissue TFA takes into account possible confounding from individual TFA, but at the same time may change the interpretation of the model estimates.

In epidemiological studies of TFA and CVD, TFA from industrial sources have been shown to be associated with increased risks, whereas TFA from ruminant sources did not exhibit such an association ${ }^{(15,16)}$. This may be due to different biological effects of specific TFA or lower intake levels of TFA from ruminant sources. However, using cubic splines to model the associations between the proportions of adipose tissue TFA and changes in anthropometry, no threshold was observed within the exposure range in the present study. Since none of the associations was significantly non-linear, we also performed linear regression analyses. These should be considered as post hoc analyses and the CI should be interpreted with reservations. However, all the linear correlation coefficients point in the same direction and do not indicate that specific TFA have different associations with changes in anthropometry.

The lack of an association between the proportions of adipose tissue TFA and changes in weight and WC in the present study is in contrast to previous findings of cohort studies using the dietary intake of TFA as an exposure variable ${ }^{(7-9)}$. In a cross-sectional study carried out by Smit et al. ${ }^{(10)}$, inverse associations were observed between total adipose tissue 18: $1 t$ and BMI and WC. The proportions of specific TFA in adipose tissue observed in the present study are similar to those reported by Smit et $a l^{(10)}$, and thus differences in exposure levels cannot explain the discrepancies between the two studies. However, different food sources of $18: 1 t$ in the Costa Rican and Danish populations may be part of the explanation, as confounding from other nutrients in the food sources contributing to the TFA intake cannot be excluded. The proportions of adipose tissue rumenic acid were not significantly associated with changes in weight or WC in the 
present study. In agreement with this, Smit et al. ${ }^{(10)}$ did not find rumenic acid to be significantly associated with BMI or WC, although they did find a positive association between rumenic acid and skinfold thickness. Rumenic acid is an isomer of CLA, but, even though animal studies have shown that feeding CLA reduces body weight in rodents, evidence for an association between CLA consumption and body composition in humans has not been established ${ }^{(5,6)}$. It was not possible to investigate the associations between nonconjugated trans-18:2 isomers and changes in weight and WC in the present study, but previously positive associations between these TFA and anthropometry have been reported in cross-sectional studies ${ }^{(10,37)}$.

The associations between adipose tissue TFA and changes in anthropometry may depend on the size of the fat mass. Due to the rather small sample size in the present study, it was not possible to investigate the associations between the adipose tissue levels of TFA and changes in anthropometry across strata of fat mass measured as BMI and WC at baseline. Sex was considered as a potential effect modifier due to sex-related differences in the size of the fat mass. The findings of the present study, however, do not suggest systematical differences in the associations across sex strata.

Potential biological mechanisms to explain the association between TFA and obesity have not been identified ${ }^{(1)}$. It has been hypothesised that TFA may lead to abdominal obesity through impairment of insulin sensitivity ${ }^{(8,38)}$, but studies of TFA and insulin sensitivity have reported mixed results ${ }^{(39)}$. In a randomised cross-over trial of diets enriched with monounsaturated fat, saturated fat or TFA, higher fat oxidation has been observed after the consumption of a TFA diet ${ }^{(40)}$, while in another trial, energy expenditure and substrate oxidation did not differ after the consumption of a large dose of monounsaturated fat, polyunsaturated fat or $\mathrm{TFA}^{(41)}$.

In conclusion, this study suggests that neither the proportions of total 18:1t nor those of specific TFA in adipose tissue are associated with subsequent changes in weight or WC within the exposure range observed in the Danish population.

\section{Supplementary material}

To view supplementary material for this article, please visit http://dx.doi.org/10.1017/S0007114513003747

\section{Acknowledgements}

The authors thank the Danish Cancer Society and the staff at the Diet, Cancer and Health study for the collection and administration of data.

The present study is part of the project Health Promoting Effect of Milk-Derived Products, which is supported by the Danish Council for Strategic Research (Contract 2101-080053) and the Danish Dairy Research Foundation, and part of the research program of the Danish Obesity Research Centre (DanORC, http://www.danorc.dk), which is supported by the Danish Council for Strategic Research (Contract 210106-0005). The Danish Council for Strategic Research and the
Danish Dairy Research Foundation had no role in the design and analysis of the study or in the writing of this article.

The authors' contributions are as follows: C. P. H., K. O. and M. U. J. contributed to the study design; A. T. and K. O. collected the data; C. P. H. carried out the statistical analyses; C. P. H., T. L. B., J. N. Ø., C. C. D., L. I. H., E. B. S., A. T., T. I. A. S., K. O. and M. U. J. were involved in the interpretation of the data and critical revision of the manuscript for important intellectual content; C. P. H. wrote the manuscript. All authors read and approved the final manuscript.

None of the authors has any conflicts of interest to declare.

\section{References}

1. Thompson AK, Minihane AM \& Williams CM (2011) Trans fatty acids and weight gain. Int J Obes (Lond) 35, 315-324.

2. Stender S, Astrup A \& Dyerberg J (2008) Ruminant and industrially produced trans fatty acids: health aspects. Food Nutr Res $\mathbf{5 2}$.

3. Turpeinen AM, Mutanen M, Aro A, et al. (2002) Bioconversion of vaccenic acid to conjugated linoleic acid in humans. Am J Clin Nutr 76, 504-510.

4. Haug A, Hostmark AT \& Harstad OM (2007) Bovine milk in human nutrition - a review. Lipids Health Dis 6, 25.

5. Gebauer SK, Psota TL \& Kris-Etherton PM (2007) The diversity of health effects of individual trans fatty acid isomers. Lipids 42, 787-799.

6. Wang Y \& Jones PJ (2004) Dietary conjugated linoleic acid and body composition. Am J Clin Nutr 79, 1153S-1158S

7. Field AE, Willett WC, Lissner L, et al. (2007) Dietary fat and weight gain among women in the Nurses' Health Study. Obesity (Silver Spring) 15, 967-976.

8. Koh-Banerjee P, Chu NF, Spiegelman D, et al. (2003) Prospective study of the association of changes in dietary intake, physical activity, alcohol consumption, and smoking with 9-y gain in waist circumference among 16587 US men. Am J Clin Nutr 78, 719-727.

9. Hansen CP, Berentzen TL, Halkjaer J, et al. (2012) Intake of ruminant trans fatty acids and changes in body weight and waist circumference. Eur J Clin Nutr 66, 1104-1109.

10. Smit LA, Willett WC \& Campos H (2010) trans-Fatty acid isomers in adipose tissue have divergent associations with adiposity in humans. Lipids 45, 693-700.

11. Tjonneland A, Overvad K, Thorling E, et al. (1993) Adipose tissue fatty acids as biomarkers of dietary exposure in Danish men and women. Am J Clin Nutr 57, 629-633.

12. Arner P, Bernard S, Salehpour M, et al. (2011) Dynamics of human adipose lipid turnover in health and metabolic disease. Nature 478, 110-113.

13. Hodson L, Skeaff CM \& Fielding BA (2008) Fatty acid composition of adipose tissue and blood in humans and its use as a biomarker of dietary intake. Prog Lipid Res 47, 348-380.

14. Willett W (1998) Nutritional Epidemiology, 2nd ed. New York, NY: Oxford University Press.

15. Bendsen NT, Christensen R, Bartels EM, et al. (2011) Consumption of industrial and ruminant trans fatty acids and risk of coronary heart disease: a systematic review and meta-analysis of cohort studies. Eur J Clin Nutr 65, 773-783.

16. Mozaffarian D, Katan MB, Ascherio A, et al. (2006) Trans fatty acids and cardiovascular disease. N Engl J Med 354, 1601-1613.

17. Tjonneland A, Olsen A, Boll K, et al. (2007) Study design, exposure variables, and socioeconomic determinants of participation in Diet, Cancer and Health: a population-based 
prospective cohort study of 57,053 men and women in Denmark. Scand J Public Health 35, 432-441.

18. Beynen AC \& Katan MB (1985) Rapid sampling and longterm storage of subcutaneous adipose-tissue biopsies for determination of fatty acid composition. Am J Clin Nutr 42, 317-322.

19. Gjerstorff ML (2011) The Danish Cancer Registry. Scand J Public Health 39, 42-45.

20. Andersen TF, Madsen M, Jorgensen J, et al. (1999) The Danish National Hospital Register. A valuable source of data for modern health sciences. Dan Med Bull 46, 263-268.

21. Carstensen B, Kristensen JK, Ottosen P, et al. (2008) The Danish National Diabetes Register: trends in incidence, prevalence and mortality. Diabetologia 51, 2187-2196.

22. Harrell FE (2001) Regression Modeling Strategies. With Applications to Linear Models, Logistic Regression and Survival Analysis, 1st ed. New York, NY: Springer.

23. Greenland S (1995) Dose-response and trend analysis in epidemiology: alternatives to categorical analysis. Epidemiology 6, 356-365.

24. Cantwell MM (2000) Assessment of individual fatty acid intake. Proc Nutr Soc 59, 187-191.

25. Hunter DJ, Rimm EB, Sacks FM, et al. (1992) Comparison of measures of fatty acid intake by subcutaneous fat aspirate, food frequency questionnaire, and diet records in a freeliving population of US men. Am J Epidemiol 135, 418-427.

26. Garland M, Sacks FM, Colditz GA, et al. (1998) The relation between dietary intake and adipose tissue composition of selected fatty acids in US women. Am J Clin Nutr 67, 25-30.

27. London SJ, Sacks FM, Caesar J, et al. (1991) Fatty acid composition of subcutaneous adipose tissue and diet in postmenopausal US women. Am J Clin Nutr 54, 340-345.

28. Baylin A, Kabagambe EK, Siles X, et al. (2002) Adipose tissue biomarkers of fatty acid intake. Am J Clin Nutr 76, 750-757.

29. Lemaitre RN, King IB, Patterson RE, et al. (1998) Assessment of trans-fatty acid intake with a food frequency questionnaire and validation with adipose tissue levels of transfatty acids. Am J Epidemiol 148, 1085-1093.

30. Leth T, Jensen HG, Mikkelsen AA, et al. (2006) The effect of the regulation on trans fatty acid content in Danish food. Atheroscler Suppl 7, 53-56.
31. Mamalakis G, Kafatos A, Manios Y, et al. (2002) Abdominal vs buttock adipose fat: relationships with children's serum lipid levels. Eur J Clin Nutr 56, 1081-1086.

32. Bigaard J, Spanggaard I, Thomsen BL, et al. (2005) Selfreported and technician-measured waist circumferences differ in middle-aged men and women. $J$ Nutr 135, 2263-2270.

33. Rosell M, Johansson G, Berglund L, et al. (2005) The relation between alcohol intake and physical activity and the fatty acids $14: 0,15: 0$ and $17: 0$ in serum phospholipids and adipose tissue used as markers for dairy fat intake. BrJ Nutr $\mathbf{9 3}$ 115-121.

34. Biong AS, Veierod MB, Ringstad J, et al. (2006) Intake of milk fat, reflected in adipose tissue fatty acids and risk of myocardial infarction: a case-control study. Eur J Clin Nutr 60, $236-244$

35. Warensjo E, Jansson JH, Cederholm T, et al. (2010) Biomarkers of milk fat and the risk of myocardial infarction in men and women: a prospective, matched case-control study. Am J Clin Nutr 92, 194-202.

36. Smedman AE, Gustafsson IB, Berglund LG, et al. (1999) Pentadecanoic acid in serum as a marker for intake of milk fat: relations between intake of milk fat and metabolic risk factors. Am J Clin Nutr 69, 22-29.

37. Lee E, Lee S \& Park Y (2008) n-3 Polyunsaturated fatty acids and trans fatty acids in patients with the metabolic syndrome: a case-control study in Korea. Br J Nutr 100, 609-614

38. Kavanagh K, Jones KL, Sawyer J, et al. (2007) Trans fat diet induces abdominal obesity and changes in insulin sensitivity in monkeys. Obesity (Silver Spring) 15, 1675-1684.

39. Micha R \& Mozaffarian D (2009) Trans fatty acids: effects on metabolic syndrome, heart disease and diabetes. Nat Rev Endocrinol 5, 335-344.

40. Lovejoy JC, Smith SR, Champagne CM, et al. (2002) Effects of diets enriched in saturated (palmitic), monounsaturated (oleic), or trans (elaidic) fatty acids on insulin sensitivity and substrate oxidation in healthy adults. Diabetes Care 25, 1283-1288.

41. Flint A, Helt B, Raben A, et al. (2003) Effects of different dietary fat types on postprandial appetite and energy expenditure. Obes Res 11, 1449-1455. 